\title{
Regional Controllability of Semi-Linear Distributed Parabolic Systems: Theory and Simulation
}

\author{
Asmae Kamal $^{1}$, Ali Boutoulout ${ }^{1}$, Sidi Ahmed Ould Beinane ${ }^{2}$ \\ ${ }^{1}$ TSI Group, Department of Mathematics and Computer Sciences, Faculty of Sciences, \\ Moulay Ismail University, Meknes, Morocco \\ ${ }^{2}$ Department of Mathematics, College of Sciences, Al Jouf University, Sakakah, KSA \\ Email: \{as2.kamal, boutouloutali\}@yahoo.fr, beinane06@gmail.com
}

Received March 20, 2012; revised May 2, 2012; accepted May 10, 2012

\begin{abstract}
The aim of this brief paper is to give several results concerning the regional controllability of distributed systems governed by semi-linear parabolic equations. We concentrate on the determination of a control achieving internal and boundary regional controllability. The approach is based on an extension of the Hilbert Uniqueness Method (HUM) and Schauder's fixed point theorem. We give a numerical example developed in internal and boundary sub region. These numerical illustrations show the efficiency of the approach and lead to conjectures.
\end{abstract}

Keywords: Semi-Linear Parabolic Systems; Regional; Internal/Boundary Controllability; Fixed-Point Theorems; Distributed System; HUM Approach

\section{Introduction}

Many scientific and engineering problems can be modeled by partial differential equations, integral equations, or coupled ordinary and partial differential equations that can be described as differential equations in infinite-dimensional spaces using semi groups. Nonlinear integrodifferential equations, with and without delays, serve as an abstract formulation for many partial integrodifferential equations which arise in problems connected with heat flow in materials with memory, viscoelasticity, and other physical phenomena. In particular, Sobolev-type equations occur in thermodynamics in the flow of fluid through fissured rocks, in the shear of second-order fluids, and in soil mechanics. So, the study of controllability results for such systems in infinite-dimensional spaces is important.

For the motivation of abstract systems and the controllability of linear systems, one can refer to the books by Curtain and Pritchard [1], and by Curtain and Zwart [2]. For an earlier survey on the controllability of nonlinear systems using fixed-point theorems, including nonlinear delays systems, see [3]. The approximate controllability of nonlinear systems when the semigroup generated by $A$ is compact has been studied also by many authors. The results of Zhou [4] and Naito [5] give sufficient conditions on $B$ with infinite-dimensional range or necessary and sufficient conditions based on more strict assumptions on B. Li and Yong [6] studied the same problem assuming the approximate controllability of the associated linear system under arbitrary perturbation in $L_{\infty}(I, L(X))$. Bian [7] investigated the approximate controllability for a class of semi-linear systems, [8] used the Banach fixed-point theorem to obtain a local exact controllability in the case of nonlinearities with small Lipschitz constants. Zhang [9] studied the local exact controllability of semi-linear evolutions systems. Naito [5] and Seidmann [10] used the Schauder fixed-point theorem to prove the invariance of the reachable set under nonlinear perturbations. Klamka [11-13] studied sufficient conditions for constrained exact controllability in a prescribed time interval for semi-linear dynamical systems in which the nonlinear term is continuously Frechet differentiable are formulated and proved assuming that the controls take values in a convex and closed cone with vertex at zero. The method used covers a wide class of semi-linear abstract dynamical systems and is specially useful for semi-linear ones with delays. Balachandran and Sakthivel [14] studied the controllability of semilinear integrodifferential systems in Banach spaces by using the Schaefer fixed-point theorem. Fabre et al. [15] prove approximate controllability in $L^{p}(\Omega)$ for $1 \leq p<$ $+\infty$ by means of a control which can be internal or on the boundary and when the nonlinearity is globally Lipschitz. Other related abstract results were given by Zuazua [16], Lasiecka et al. [17] and Kassara et al. [18].

The study of various analytic concepts related to controllability and stability of such systems is, in general, 
delicate and considering only linear model can not be sufficient in particular when some properties of system needs to be satisfied only in some part of the system evolution domain. From practical point of view, it is very natural to consider the analysis of such systems only in some subregion of its evolution system domain. This is the aim of regional analysis.

The regional analysis of distributed parameter system has recieved an intensive study in the last three decades.

The term "regional analysis" has been used to refer to control problems in which the target of our interest is not fully specified as a state, but refers only to a smaller region $\omega$ of the system domain. This concept has been widely developed and interesting results have been obtained, in particular, the possibility to reach a state only on an internal subregion $\omega$ of $\Omega$ (El Jai et al. [19]) or on a part of the boundary $\partial \Omega$ of (Zerrik et al. [20]). The principal reason for introducing this concept is that, first it makes sense for the usual controllability concept closer to real world problem and, second, it can be applied to systems which are not controllable on the whole domain. Here we are interested on regional controllability of semi-linear parabolic systems. More precisely the question concerns the possibility of regional controllability for semi-linear system in the case where the desired state is given only on an internal subregion $\omega$ of $\Omega$ or on a part of the boundary $\partial \Omega$ of $\Omega$.

The interest of this work focused on the development of an approach that leads to numerical implementation for the computation of the control which steers the system from an initial state to a given regional internal and boundary state. A typical motivating example is the case of a biological reactor, where the problem is to regulate the concentration of a susbstratum at the bottom of the reactor [21].

In Section 2, first we present some preliminary material and state internal regional controllability problem of semi-linear systems. Next, we concentrate on the determination of a control achieving regional internal controllability, and we develop a numerical approach that leads to a useful algorithm and successfully tested through a diffusion process. Section 3 is focused on the regional boundary target control problem, and an approach is developed that leads to a numerical algorithm for the computation of a control which achieves regional boundary controllability. Numerical illustrations show the efficiency of the approach and lead to conjectures.

\section{Regional Internal Controllability}

\subsection{Statement of the Problem}

Let $\Omega$ be a regular bounded open set of $\operatorname{IR}^{\mathrm{n}}, n \geq 1$, with boundary $\partial \Omega$. For a given time $T>0$, let $Q=\Omega \times[0, T]$, and $\Sigma=\partial \Omega \times[0, T]$. We consider a semi-linear parabolic system excited by controls which can be applied via various types of actuators given by the following equation

$$
\begin{cases}\frac{\partial y}{\partial t}(x, t)=A y(x, t)+N y(x, t)+B u(t) & Q \\ y(\xi, t)=0 & \Omega \\ y(x, 0)=y_{0}(x) & \Omega\end{cases}
$$

where $A$ is a second-order linear differential operator, which generates a strongly continuous semi-group $(S(t))_{t \geq 0}$ on Hilbert space $L^{2}(\Omega)$ and $N$ a locally lipschitz continuous nonlinear operator. $B \in \mathcal{L}\left(I R^{p}, L^{2}(\Omega)\right)$, $y_{0} \in L^{2}(\Omega)$ and $u \in \tilde{U}$ where

$$
\tilde{U}=\left\{u \in L^{2}\left(0, T ; I R^{p}\right) \mid y_{u}(T) \in L^{2}(\Omega)\right\} ;
$$

where $p$ represents the number of actuators. We denote by $U$ the completion of the space $\tilde{U}$ endowed with the standard norm of $L^{2}(0, T ; \Omega)$. Denote by $y_{u}($.$) the$ solution of (2.1) when it is excited by a control $u$, suppose that $y_{u}(.) \in L^{2}\left(0, T ; L^{2}(\Omega)\right)$. Let us recall that an actuator is conventionally defined by a couple $(D, f)$, where $D \subset \bar{\Omega}$ is the geometric support of the actuator and $f$ is the spatial distribution of the action on the support $D$, see [22]. In this case, $B u(t)=\chi_{D} f(x) u(t)$. In the case of pointwise actuator (internal or boundary), $D=\{b\}$ and $f=\delta_{b}$, where $\delta_{b}$ is the Dirac mass concentrated at $b$; in this case, the actuator is denoted by $\left(b, \delta_{b}\right)$ and $B u(t)=\delta_{b}(x) u(t)$.

Let $y_{u}$ be the solution of (2.1) excited a control $u$ and assume that $y_{u}(.) \in L^{2}\left(0, T ; L^{2}(\Omega)\right)$, see [23].

For $\omega \subset \Omega$, open, nonempty and of positive Lebesgue measure, we consider the operator restriction

$$
\begin{gathered}
\chi_{\omega}: L^{2}(\Omega) \rightarrow L^{2}(\omega) \\
y \rightarrow y_{\mid \omega}
\end{gathered}
$$

and $\chi_{\omega}^{*}$ denotes the adjoint operator.

\section{Definition 1}

- System (2.1) is said to be $\omega$-exactly regionally controllable if for all $y_{d} \in L^{2}(\omega)$, there exists a control $u \in U$ such that $\chi_{\omega} y_{u}(T)=y_{d}$.

- $\operatorname{System}(2.1)$ is said to be $\omega$-approximately regionally controllable if for all $y_{d} \in L^{2}(\omega)$ and for all $\varepsilon>0$, there exists a control $u \in U$ such that

$$
\left\|\chi_{\omega} y_{u}(T)-y_{d}\right\|_{L^{2}(\omega)} \leq \varepsilon
$$

The notion of regional controllability considered as a particular case of output controllability was introduced and developed for linear system in (E1 Jai et al. [19], Zerrik et al. [20]).

It is clear that:

- If system (2.1) is regionally controllable on $\omega$ then it is regionally controllable on any $\omega_{1} \subset \omega$. 
- In the linear case, one can find states which are approximately regionally controllable on $\omega$ but not controllable on the whole domain $\Omega$, see $[19,22]$.

To study the controllability of the system (2.1), we consider its corresponding linear system $[N \equiv 0$ in (2.1)],

$$
\begin{cases}\frac{\partial y}{\partial t}(x, t)=A y(x, t)+B u(t) & Q \\ y(\xi, t)=0 & \Sigma \\ y(x, 0)=y_{0}(x) & \Omega\end{cases}
$$

The problem of regional controllability on $\omega$ for (2.1) can be stated as follows:

\section{Problem}

$$
\left\{\begin{array}{l}
\text { For } y_{d} \text { a desired state, find a control } \\
u \in U \text { such that } \chi_{\omega} y_{u}(T)=y_{d}
\end{array}\right.
$$

More precisely, it is asked to find a control which steers system (2.1), at time $T$, to a desired state defined in subregion $\omega \subset \Omega$.

\subsection{Hilbert Uniqueness Approach}

The aim of this section is to give an extension of regional controllability and Hilbert uniqueness method introduced in the linear case by (El Jai et al. [19]) and [24] which allows the characterization of a control $u^{*}$ solution of (2.3). The system (2.2) is approximately controllable in $\omega$ and system $(2.1)$ is excited by a zone actuator $(D, f)$. System (2.1) may be rewritten in the form

$$
\begin{cases}\frac{\partial y}{\partial t}(x, t)=A y(x, t)+N y(x, t)+\chi_{D} f(x) u(t) & Q \\ y(\xi, t)=0 & \Sigma \\ y(x, 0)=y_{0}(x) & \Omega\end{cases}
$$

and the operator $N: L^{2}\left(0, T ; L^{2}(\Omega)\right) \rightarrow L^{2}\left(0, T ; L^{2}(\Omega)\right)$ verify

$$
\begin{aligned}
& \|N(x)\|_{L^{2}\left(0, T ; L^{2}(\Omega)\right)} \leq C\|x\|_{L^{2}\left(0, T ; L^{2}(\Omega)\right)}, \\
& C=c(\Omega, T)
\end{aligned}
$$

Let $G=\left\{g \in L^{2}(\Omega)\right.$ such that $g=0$ on $\left.\Omega \backslash \omega\right\}$

$$
\begin{cases}\frac{\partial \varphi}{\partial t}(x, t)=-A^{*} \varphi(x, t) & Q \\ \varphi(\xi, t)=0 & \Sigma \\ \varphi(x, T)=\varphi_{0}(x) & \Omega\end{cases}
$$
[25].

Which has a unique solution $\varphi \in L^{2}\left(0, T ; L^{2}(\Omega)\right)$ see

For a given $\varphi_{0} \in G$, we consider the system (2.6) and define the mapping

$$
\left\|\varphi_{0}\right\|_{G}=\left(\int_{0}^{T}\langle f, \varphi(t)\rangle_{L^{2}(D)}^{2} \mathrm{~d} t\right)^{\frac{1}{2}}
$$

Which is a norm on $G$; since the system (2.2) is approximately controllable in $\omega$.

Consider the system

$$
\begin{cases}\frac{\partial y}{\partial t}(x, t)=A y(x, t)+N y(x, t)+\langle\varphi(t), f\rangle_{L^{2}(D)} \chi_{D} f(x) & Q \\ y(\xi, t)=0 & \Omega \\ y(x, 0)=y_{0}(x) & \Omega\end{cases}
$$

and the associated linear system

$$
\begin{cases}\frac{\partial y}{\partial t}(x, t)=A y(x, t)+\langle\varphi(t), f\rangle_{L^{2}(D)} \chi_{D} f(x) & Q \\ y(\xi, t)=0 & \Omega \\ y(x, 0)=y_{0}(x) & \Omega\end{cases}
$$

The system (2.8) may be decomposed in the following three systems

$$
\begin{cases}\frac{\partial \Psi_{1}}{\partial t}(x, t)=A \Psi_{1}(x, t) & Q \\ \Psi_{1}(\xi, t)=0 & \Sigma \\ \Psi_{1}(x, 0)=y_{0}(x) & \Omega\end{cases}
$$

and 


$$
\begin{cases}\frac{\partial \Psi_{2}}{\partial t}(x, t)=A \Psi_{2}(x, t)+\langle\varphi(t), f\rangle_{L^{2}(D)} \chi_{D} f(x) & Q \\ \Psi_{2}(\xi, t)=0 & \Omega \\ \Psi_{2}(x, 0)=y_{0}(x) & \Omega\end{cases}
$$

where $\varphi$ is the solution of (2.6) and

$$
\begin{cases}\frac{\partial \Psi_{3}}{\partial t}(x, t)=A \Psi_{3}(x, t)++N\left(\Psi_{1}+\Psi_{2}+\Psi_{3}\right) & Q \\ \Psi_{3}(\xi, t)=0 & \Sigma \\ \Psi_{3}(x, 0)=y_{0}(x) & \Omega\end{cases}
$$

We denote the completion of the set $G$ with respect to the norm (2.7) again by $G$.

Let $\wedge: G \rightarrow G^{*}$ be defined by $\wedge\left(\varphi_{0}\right)=P\left(\Psi_{2}(T)\right)$ where $P=\chi_{\omega}^{*} \chi_{\omega}$.

Now, with the nonlinear operator

$$
\mu \varphi_{0}=P\left(\Psi_{2}(T)\right)+P\left(\Psi_{3}(T)\right)
$$

The problem of regional controllability (2.3) turns up to solve the equation $\mu \varphi_{0}=\chi_{\omega}^{*} y_{d}-P\left(\Psi_{1}(T)\right)$ which is equivalent to

$$
\begin{aligned}
P\left(\Psi_{2}(T)\right) & =\chi_{\omega}^{*} y_{d}-P\left(\Psi_{3}(T)\right)-P\left(\Psi_{1}(T)\right) \\
& =\chi_{\omega}^{*} y_{d}-K\left(\varphi_{0}\right)-P\left(\Psi_{1}(T)\right)
\end{aligned}
$$

where $K: G \rightarrow G^{*}$ is the operator defined by the formula $K\left(\varphi_{0}\right)=P\left(\Psi_{3}(T)\right)$. Then we have

$$
\chi_{\omega}^{*} y_{d}-K\left(\varphi_{0}\right)-P\left(\Psi_{1}(T)\right)=\wedge \varphi_{0}
$$

The linear system (2.9) is approximately regionally controllable in $\omega$, then $\wedge$ is one to one see [19].

Apply $\wedge^{-1}$ the equation (2.13), we have

$$
\wedge^{-1}\left(\chi_{\omega}^{*} y_{d}\right)-\wedge^{-1} K\left(\varphi_{0}\right)-\wedge^{-1} P\left(\Psi_{1}(T)\right)=\varphi_{0}
$$

Now, we define the nonlinear operator $\tilde{K}$ by

$\tilde{K}\left(\varphi_{0}\right)=\wedge^{-1}\left(\chi_{\omega}^{*} y_{d}\right)-\wedge^{-1} K\left(\varphi_{0}\right)-\wedge^{-1} P\left(\Psi_{1}(T)\right)$

Then the problem (2.3) of system (2.7) turns up to search a fixed point of $\tilde{K}$, then we have

\section{Proposition 1}

Assume that (2.5) holds. If the linear system (2.9) is approximately regionally controllable in $\omega$, then the control $u^{*}(t)=\langle\varphi(t), f\rangle_{L^{2}(D)}$ steers the system (2.8) to $y_{d}$ in $\omega$ at $t=T$, where $\varphi$ is the solution of the system (2.6) and $\varphi_{0}$ is a fixed point of the operator $\tilde{K}$ given by (2.14).

Sketch of the proof: The proof may be easily achieved with the following two steps:

Step 1: We prove that $K$ is a compact operator and then deduce that $\tilde{K}$ is also compact.

Step 2: Applying the Schauder fixed-point theorem, we see that the operator $\tilde{K}$ has a fixed point. For more details we refer the reader to [26].

\section{Remark 1}

The above approach is a generalization of the Hilbert uniqueness method given in the linear case $(N \equiv 0)$ and the operator $\mu$ coincides with the isomorphism $\wedge$.

\section{Algorithm 1}

Summing up, in the zone case, the regional controllability is obtained via the following simplified algorithm

- Step 1: We take the following initial conditions $\omega$, $y_{d}, f, D$ and $\varepsilon$.

- Step 2: Using the pseudo-code.

- Resolution of (2.6) and obtaining $\varphi$.

- Resolution of (2.10) and obtaining $\Psi_{1}$.

- Resolution of (2.11) and obtaining $\Psi_{2}$.

- Resolution of (2.12) and obtaining $\Psi_{3}$.

- Calculation of $\wedge^{-1}$ and obtaining $\tilde{K}\left(\varphi_{0}\right)$.

- Resolution of $\varphi_{0}=K\left(\varphi_{0}\right)$ and obtaining $\varphi_{0}$.

- Until $\left\|\varphi_{0}-K\left(\varphi_{0}\right)\right\| \leq \varepsilon$.

- Step 3: The control $u^{*}(t)=\langle\varphi(t), f\rangle_{L^{2}(D)}$.

\subsection{Simulations}

The goal of this section is to test the efficiency of the previous algorithm. The obtained results are related to the considered subregion, the desired state and the actuator structure. Let $\Omega=[0,1] \times[0,1]$, and consider the one-dimensional diffusion system described by

$$
\begin{cases}\frac{\partial y}{\partial t}(x, t)=(0.1) \frac{\partial^{2} y}{\partial x^{2}}(x, t)+B u(t)+\sum_{j=1}^{\infty}\left|\left\langle y, \varphi_{j}\right\rangle\right|\left\langle y, \varphi_{j}\right\rangle \varphi_{j} & {[0,1] \times[0, T]} \\ y(0, t)=y(1, t)=0 & {[0, T]} \\ y(x, 0)=0 & {[0,1]}\end{cases}
$$




\subsubsection{Zone Actuator}

In this case $B u(t)=\chi_{D}(x) u(t)$ where $D=[0.22,0.37]$ The subregion under consideration is $\omega=[0.30,0.58]$.

Let $y_{d}(x)=-x(1-x)(x-0.9)(x-0.4) / 10$ be the desired regional state in $\omega$. Using the previous algorithm 1 , the simulation gives the Figure 1.

The regional desired state $y_{d}$ is reached with error

$$
\left\|\chi_{\omega} y_{u^{*}}(T)-y_{d}\right\|_{L^{2}(\omega)}^{2}=7.16 \times 10^{-10}
$$

and transfer cost $\left\|u^{*}\right\|^{2}=1.57 \times 10^{-1}$.

\subsubsection{Pointwise Actuator}

In this case $B u(t)=\delta_{b} u(t)$ where $b=0.67$.

We consider the subregion $\omega=[0.38,0.68]$.

Let $y_{d}(x)=2 x(1-x)(x-0.9)(x-0.4)$ be the desired regional state on $\omega$. The simulation gives the Figure 2 .

The regional desired state $y_{d}$ is reached with error

$$
\left\|\chi_{\omega} y_{u^{*}}(T)-y_{d}\right\|_{L^{2}(\omega)}^{2}=1.15 \times 10^{-9}
$$

and transfer cost $\left\|u^{*}\right\|^{2}=8.75 \times 10^{-2}$.

\subsubsection{Relation between the Subregion and Location of the Pointwise Actuator}

The following simulation results show the evolution of the desired state error with respect to the actuator location. Figure 3 shows that:

- For a given subregion $\omega$ and a desired state, there is an optimal actuator location (optimal in the sense that it leads to a solution which is very close to the desired state).

- When the actuator is located sufficiently far from the subregion $\omega$, the estimated state error is constant for any location.

- The worst locations correspond to non strategic actuators in $\omega=[0,1]$, as developed in the linear case see [19].

Figure 4 shows that, for a given subregion and a desired state, there is an optimal actuator location in the sense that it leads to a smaller transfer cost.

The results are similar for other types of actuators.

\section{Regional Boundary Controllability}

The aim of this section is to give an extension of the concepts of regional internal controllability [26] to the case where is a part of the boundary of the domain. The developed $\omega$ method is original and leads to a numerical algorithm illustrated by simulations.

\subsection{Considered System and Problem Statement}

Let $\Omega$ be a bounded open domain in $\operatorname{IR}^{\mathrm{n}}(\mathrm{n}=1,2,3)$ with a regular boundary $\partial \Omega$. For $T>0$, we write

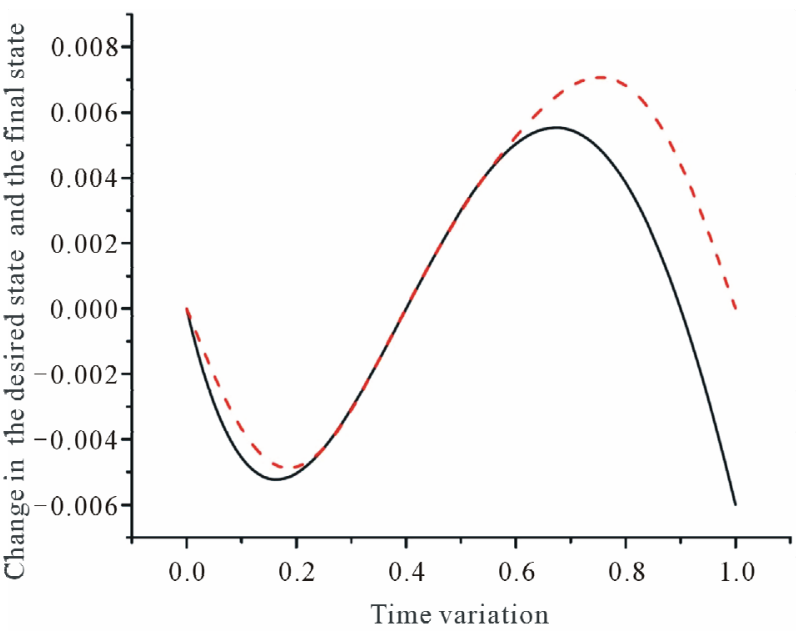

Figure 1. Desired state (continuous line) and final state (dashed line) on the region $\omega$.

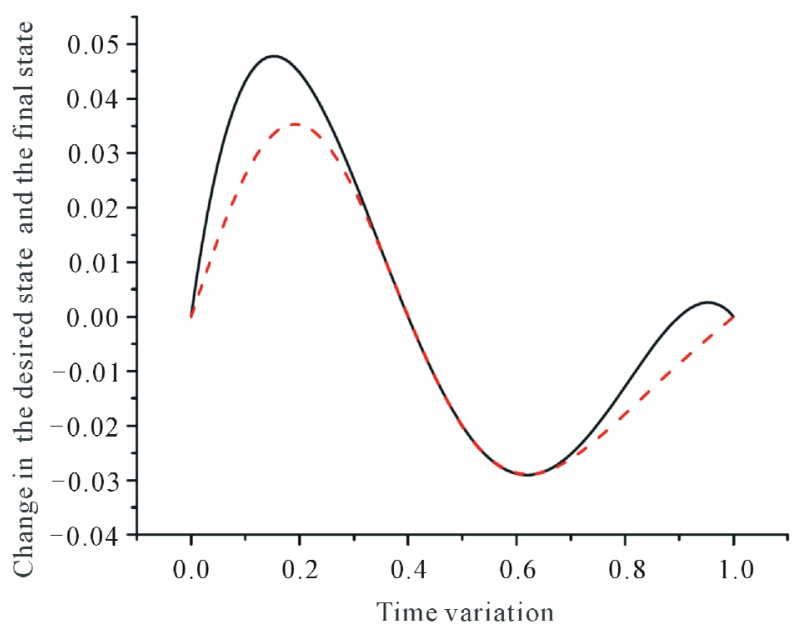

Figure 2. Desired state (continuous line) and final state (dashed line) on the region $\omega$.

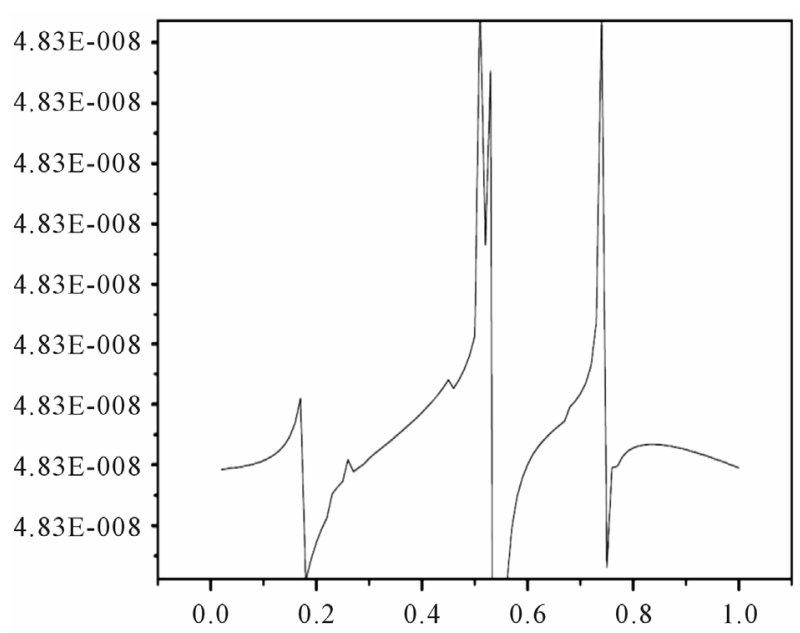

Figure 3. The evolution of the estimated state error with respect to the actuator locations. 


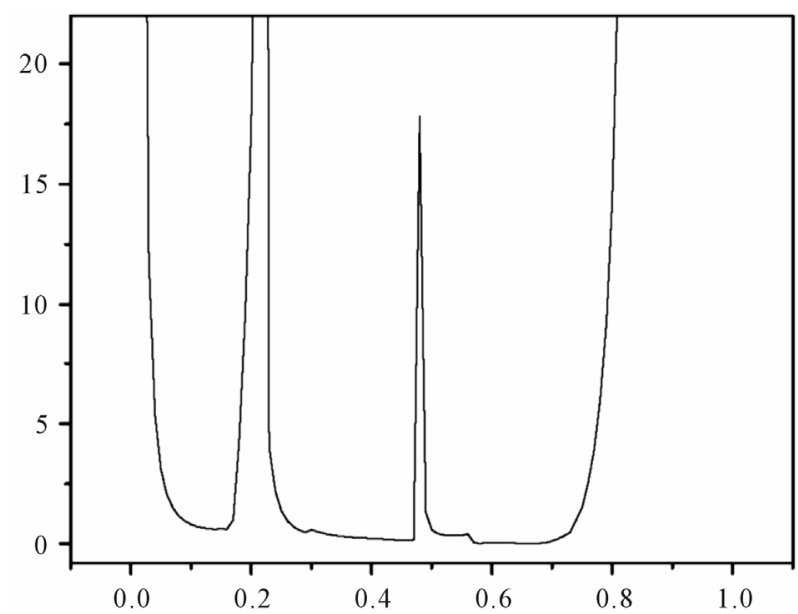

Figure 4. The evolution of the transfer cost with respect to the actuator locations.

$Q=\Omega \times[0, T], \quad \sum=\partial \Omega \times[0, T]$ and consider the following semi-linear parabolic system

$$
\begin{cases}\frac{\partial y}{\partial t}(x, t)=A y(x, t)+N y(x, t)+B u(t) & Q \\ \frac{\partial y}{\partial v_{A}}(\xi, t)=0 & \Sigma \\ y(x, 0)=y_{0}(x) & \Omega\end{cases}
$$

where

- $A$ is a second-order linear differential operator, which generates a strongly continuous semi-group $(S(t))_{t \geq 0}$ on Hilbert space $X=H^{1}(\Omega)$.

- $\quad N$ a locally lipschitz continuous nonlinear operator.

- $B \in \mathcal{L}\left(I^{p}, X\right), y_{0} \in X$.

- $u \in \tilde{U}$ where $\tilde{U}=\left\{u \in L^{2}\left(0, T ; I R^{p}\right) \mid y_{u}(T) \in X\right\}$ with $y_{u}$ be the solution of (3.1) excited by a control $u$.

We denote by $U$ the completion of the space $\tilde{U}$ endowed with the standard norm of $L^{2}\left(0, T ; \mathbb{R}^{p}\right)$.

Assume that $y_{u}(.) \in L^{2}(0, T ; X)$. The controls may be applied via various types of actuators see [22].

The associated linear system is

$$
\begin{cases}\frac{\partial y}{\partial t}(x, t)=A y(x, t)+B u(t) & Q \\ \frac{\partial y}{\partial v_{A}}(\xi, t)=0 & \Sigma \\ y(x, 0)=y_{0}(x) & \Omega\end{cases}
$$

For $\Gamma$ being a regular subset of $\partial \Omega$ which has positive Lebesgue measure, consider the restriction operator

$$
\begin{gathered}
\chi_{\Gamma}: H^{\frac{1}{2}}(\partial \Omega) \rightarrow H^{\frac{1}{2}}(\Gamma) \\
\left.y \rightarrow y\right|_{\Gamma}
\end{gathered}
$$

where $\chi_{\Gamma}^{*}$ denotes its adjoint operator.

Let us $\gamma_{0}: H^{1}(\Omega) \rightarrow H^{\frac{1}{2}}(\partial \Omega)$ whilst $\gamma_{0}^{*}$ is considered for the adjoint operator.

We introduce the definition.

\section{Definition 2}

The system (3.1) is said to be $\Gamma$-exactly (resp. $\Gamma$ approximately) regionally controllable if for all $y_{d} \in H^{\frac{1}{2}}(\Gamma)$ (resp. for all $\varepsilon>0$ ) there exists a control $u \in U$ such that $\chi_{\Gamma} \gamma_{0} y_{u}(T)=y_{d} \quad$ (resp. $\left.\left\|\chi_{\Gamma} \gamma_{0} y_{u}(T)-y_{d}\right\|_{H^{\frac{1}{2}}(\Gamma)} \leq \varepsilon\right)$.

This definition generalizes the standard ones of exact and approximate controllability on the whole domain $\Omega$.

\section{Remark 2}

1) The notion of regional controllability considered as a particular case of output controllability was introduced and developed for linear system in [20].

2) A system which is $\Gamma$-exactly (resp. $\Gamma$-approximately) regionally controllable is $\Gamma_{1}$-exactly (resp. $\Gamma_{1}$ approximately) regionally controllable for all $\Gamma_{1} \subset \Gamma$.

3) The above definitions do not allow for pointwise or boundary controls since, for such.

4) systems $B \notin \mathcal{L}\left(I R^{p}, X\right)$ and the solution $y_{u}(.) \notin L^{2}(\Omega)$. However, the extension can be carried out in a similar manner if one takes regular controls such that $y_{u}(T) \in H^{1}(\Omega) \quad[27]$.

In the sequel, we explore the possibility of finding a control which ensues the transfer of system (3.1) to desired $y_{d}$ on the boundary subregion $\Gamma \subset \partial \Omega$, consider the problem

$$
\left\{\begin{array}{l}
\text { For } y_{d} \text { desired state, fined a control } \\
u \in U \text { such that } \chi_{\Gamma} \gamma_{0} y_{u}(T)=y_{d}
\end{array}\right.
$$

\subsection{Theoretical Approach}

Firstly, the following result provides a link between regional internal controllability see [26] and regional boundary controllability for semi-linear systems.

Consider the linear and continuous extension operator $R: H^{\frac{1}{2}}(\partial \Omega) \rightarrow H^{1}(\Omega)$ such that $\gamma_{0} R g=g$, for all $g \in H^{\frac{1}{2}}(\partial \Omega)$. For $y_{d} \in H^{\frac{1}{2}}(\Gamma)$, we denote by $\bar{y}_{d}$ the extension of $y_{d}$ to $\partial \Omega$ and we define

$$
\begin{aligned}
& D=\left\{R \bar{y}_{d} \in H^{1}(\Omega) \mid y_{d} \in H^{\frac{1}{2}}(\Gamma)\right\} \\
& V=\underset{y_{d} \in H^{\frac{1}{2}}(\Gamma)}{\cup} \operatorname{suppR}_{d} \bar{y}_{d}
\end{aligned}
$$

Let $r>0$ integer small, we set $F_{r}=\cup_{z \in \Gamma} B(z, r)$ and 
$\omega_{r}=F_{r} \cap V$, where $B(z, r)$ is the open ball of radius $r$ and center $z$, see [28]. Then, we have the following result.

Proposition 2

If the system (3.1) is $\omega_{r}$-exactly (resp. $\omega_{r}$-approximately) regionally controllable, then it is $\Gamma$-exactly (resp.

$\Gamma$-approximately) regionally controllable.

\section{Proof}

Let $y_{d} \in H^{\frac{1}{2}}(\Gamma)$, then by trace theorem, there exists $R \bar{y}_{d} \in H^{1}(\Omega)$ with a bounded support such that

$$
\gamma_{0}\left(R \bar{y}_{d}\right)=\bar{y}_{d} .
$$

Since the system (3.1) is $\omega_{r}$-exactly controllable, then there exists a control $u \in U$ such that

$$
\chi_{\omega_{r}} y_{u}(T)=\chi_{\omega_{r}} R \bar{y}_{d} .
$$

Thus $\gamma_{0}\left(\chi_{\omega_{r}} y_{u}(T)\right)=\bar{y}_{d}$ and then

$\chi_{\Gamma}\left(\gamma_{0}\left(\chi_{\omega_{r}} y_{u}(T)\right)=y_{d}\right.$. Consequently, the system (3.1) is $\Gamma$-exactly controllable.

Now, if the system (3.1) is $\omega_{r}$-approximately controllable, for all $\varepsilon>0$, there exists $u \in U$ such that

$$
\left\|\chi_{\omega_{r}} y_{u}(T)-\chi_{\omega_{r}} R \bar{y}_{d}\right\|_{H^{1}\left(\omega_{r}\right)} \leq \varepsilon
$$

and by continuity of the trace mapping $\gamma_{0}$, we have

$$
\left\|\gamma_{0} \chi_{\omega_{r}} y_{u}(T)-\gamma_{0} \chi_{\omega_{r}} R \bar{y}_{d}\right\|_{H^{\frac{1}{2}}\left(\partial \omega_{r}\right)} \leq \varepsilon
$$

therefore

$$
\left\|\chi_{\Gamma} \gamma_{0} \chi_{\omega_{r}} y_{u}(T)-y_{d}\right\|_{H^{\frac{1}{2}}(\Gamma)} \leq \varepsilon
$$

Consequently, the system (3.1) is $\Gamma$-approximately controllable.

Secondly, we develop an approach devoted to characterize a control $u^{*}$ solution of problem (3.3), when the system (3.1) is $\omega_{r}$-approximately controllable. The approach we shall use is based on an extension of regional controllability techniques for linear systems developed in (El Jai et al. [19]) and Hilbert uniqueness method see [24].

The system (3.2) is excited by a control applied by means of a zone actuator $(D, f)$ where $D \neq \varnothing \subset \Omega$ is the actuator support and $f \neq 0 \in L^{2}(D)$ defines the spatial distribution of the control on $\mathrm{D}$, then the system (3.2) may be written in the form

$$
\begin{cases}\frac{\partial y}{\partial t}(x, t)=A y(x, t)+N y(x, t)+\chi_{D} f(x) u(t) & Q \\ \frac{\partial y}{\partial v_{A}}(\xi, t)=0 & \Omega \\ y(x, 0)=y_{0}(x) & \Omega\end{cases}
$$

The operator $N: L^{2}(0, T ; X) \rightarrow L^{2}(0, T ; X)$ verify

$$
\begin{aligned}
& \|N(x)\|_{L^{2}(0, T ; X)} \leq c\|x\|_{L^{2}(0, T ; X)}, \\
& 0<c=c(\Omega, T) \leq 1 \text { is a constant. }
\end{aligned}
$$

Let $G$ be the set

$$
G=\left\{g \in X \text { such that } g=0 \text { in } \Omega \backslash \omega_{r}\right\}
$$

For $y_{d} \in H^{\frac{1}{2}}(\Gamma)$, we denote by $\bar{y}_{d}$ the extension of $y_{d}$ to $\partial \Omega$.

Consider the system

$$
\begin{cases}\Delta z=0 & \Omega \\ z=\bar{y}_{d} & \partial \Omega\end{cases}
$$

where $\Delta$ is the Laplace operator. The system (3.6) has a unique solution $z$ in $H^{1}(\Omega)$. Let $z_{d}$ the restriction of $z$ in $H^{1}\left(\omega_{r}\right)$.

The problem of reaching $y_{d}$ on $\Gamma$ may then be solved by reaching $z_{d}$ on $\omega_{r}$. Then the problem (3.3) is formulated as follows:

$$
\left\{\begin{array}{l}
\text { For } z_{d} \in H^{1}\left(\omega_{r}\right), \text { a desired state, find a control } \\
u \in U \text { such that } \chi_{\omega_{r}}\left(y_{u}(T)\right)=z_{d} ?
\end{array}\right.
$$

For $\varphi_{0} \in G$, the system

$$
\begin{cases}\frac{\partial \varphi_{r}}{\partial t}(x, t)=-A^{*} \varphi_{r}(x, t) & Q \\ \frac{\partial \varphi_{r}}{\partial v_{A^{*}}}(\xi, t)=0 & \Sigma \\ \varphi_{r}(x, T)=\varphi_{0}(x) & \Omega\end{cases}
$$

has a unique solution $\varphi_{r} \in L^{2}(0, T ; X) \cap C^{0}\left(0, T ; L^{2}(\Omega)\right)$ [28].

In $G$, we define the mapping

$$
\left\|\varphi_{0}\right\|_{G}=\left(\int_{0}^{T}\left\langle f, \varphi_{r}(t)\right\rangle_{L^{2}(D)}^{2} \mathrm{~d} t\right)^{1 / 2}
$$

which is a norm on $G$; since the system is $\omega_{r}$-approximately controllable see [19].

Consider the system

$$
\begin{cases}\frac{\partial y}{\partial t}(x, t)=A y(x, t)+N y(x, t)+\left\langle\varphi_{r}(t), f\right\rangle_{L^{2}(D)} \chi_{D} f(x) & Q \\ \frac{\partial y}{\partial v_{A}}(\xi, t)=0 & \Sigma \\ y(x, 0)=y_{0}(x) & \Omega\end{cases}
$$


and its associated linear system is

$$
\begin{cases}\frac{\partial y}{\partial t}(x, t)=A y(x, t)+\left\langle\varphi_{r}(t), f\right\rangle_{L^{2}(D)} \chi_{D} f(x) & Q \\ \frac{\partial y}{\partial v_{A}}(\xi, t)=0 & \Sigma \\ y(x, 0)=y_{0}(x) & \Omega\end{cases}
$$

The system (3.10) may be decomposed into the following three systems

$$
\begin{cases}\frac{\partial \Psi_{1}}{\partial t}(x, t)=A \Psi_{1}(x, t) & Q \\ \frac{\partial \Psi_{1}}{\partial v_{A}}(\xi, t)=0 & \Sigma \\ \Psi_{1}(x, 0)=y_{0}(x) & \Omega\end{cases}
$$

and

$$
\begin{cases}\frac{\partial \Psi_{2}}{\partial t}(x, t)=A \Psi_{2}(x, t)+\left\langle\varphi_{r}(t), f\right\rangle_{L^{2}(D)} \chi_{D} f(x) & Q \\ \frac{\partial \Psi_{2}}{\partial v_{A}}(\xi, t)=0 & \Sigma \\ \Psi_{2}(x, 0)=0 & \Omega\end{cases}
$$

where $\varphi_{r}$ is the solution of (3.8) and

$$
\begin{cases}\frac{\partial \Psi_{3}}{\partial t}(x, t)=A \Psi_{3}(x, t)+N\left(\Psi_{1}+\Psi_{2}+\Psi_{3}\right) & Q \\ \frac{\partial \Psi_{3}}{\partial v_{A}}(\xi, t)=0 & \Sigma \\ \Psi_{3}(x, 0)=0 & \Omega\end{cases}
$$

We denote the completion of the set $G$ with respect to the norm (3.9) again by $G$.

Consider the operator $\wedge: G \rightarrow G^{*}$ defined by $\wedge\left(\varphi_{0}\right)=P\left(\Psi_{2}(T)\right)$ where $G^{*}$ is the dual of $G$ and $P=\chi_{\omega_{r}}^{*} \chi_{\omega_{r}}$.

Let us now define the nonlinear operator

$$
\mu \varphi_{0}=P\left(\Psi_{2}(T)\right)+P\left(\Psi_{3}(T)\right)
$$

The problem of regional controllability (3.3) turns up to solve the equation

$$
\mu \varphi_{0}=\chi_{\omega_{r}}^{*} z_{d}-P\left(\Psi_{1}(T)\right)
$$

which is equivalent to

$$
\begin{aligned}
P\left(\Psi_{2}(T)\right) & =\chi_{\omega_{r}}^{*} z_{d}-P\left(\Psi_{3}(T)\right)-P\left(\Psi_{1}(T)\right) \\
& =\chi_{\omega_{r}}^{*} z_{d}-K_{r}\left(\varphi_{0}\right)-P\left(\Psi_{1}(T)\right)
\end{aligned}
$$

where $K_{r}: G \rightarrow G^{*}$ is the operator defined by $K_{r}\left(\varphi_{0}\right)=$ $P\left(\Psi_{3}(T)\right)$, which gives

$$
\chi_{\omega_{r}}^{*} z_{d}-K_{r}\left(\varphi_{0}\right)-P\left(\Psi_{1}(T)\right)=\wedge\left(\varphi_{0}\right)
$$

Since the linear system (3.11) is $\omega_{r}$-approximately regionally controllable, then $\wedge$ is one to one see (El Jai et al. [19]).

Apply $\wedge^{-1}$ the equation (3.15), we have

$$
\wedge^{-1}\left(\chi_{\omega_{r}}^{*} z_{d}\right)-\wedge^{-1} K_{r}\left(\varphi_{0}\right)-\wedge^{-1} P\left(\Psi_{1}(T)\right)=\varphi_{0}
$$

Then a solution of problem (3.3) of system (3.10) turns up to search a fixed point of nonlinear operator $\tilde{K}_{r}$ define by

$$
\tilde{K}_{r}\left(\varphi_{0}\right)=\wedge^{-1}\left(\chi_{\omega_{r}}^{*} z_{d}\right)-\wedge^{-1} K_{r}\left(\varphi_{0}\right)-\wedge^{-1} P\left(\Psi_{1}(T)\right)
$$

Then we have:

\section{Proposition 3}

If the linear system (3.11) is $\omega_{r}$-approximately regionally controllable, then the control

$u_{r}^{*}(t)=\left\langle\varphi_{r}(t), f\right\rangle_{L^{2}(D)}$ drives the system (3.10) to $y_{d}$ in $\Gamma$ at $t=T$, where $\varphi_{r}$ is the solution of the system (3.9) and $\varphi_{0}$ is a fixed point of the operator $\tilde{K}_{r}$ given by (3.16).

Proof

Step 1: We prove that $\tilde{K}_{r}$ is a compact operator.

Let the ball $B_{p}=B(0, p)$ in $X$, we have

$$
K_{r}\left(B_{p}\right)=\left\{P\left(\Psi_{3}(T)\right) \mid \varphi_{0} \in B_{p}\right\}
$$

and we set

$$
\tilde{K}_{r}\left(B_{p}\right)=\left\{P\left(\Psi_{3}(T)\right) \mid \varphi_{0} \in B_{p}, t \in[0, T]\right\}
$$

Where $\Psi_{3}($.$) is solution of the system (3.14).$

We have

$$
\Psi_{3}(t)=\int_{0}^{t} S(t-\tau) N\left(\Psi_{1}(\tau)+\Psi_{2}(\tau)+\Psi_{3}(\tau)\right) \mathrm{d} \tau
$$

$\Psi_{3} \in C(0, T ; X)$ see [23] and there exists $c_{1}>0$ such that

$$
\left\|P\left(\Psi_{3}(T)\right)\right\|_{G^{*}} \leq c_{1}\left\|\Psi_{3}(t)\right\|_{X} .
$$

Since $S($.$) is a strongly continuous semi-group on$ $[0, T]$, then there exists $M>0$ such that

$$
\|S(t)\|_{L(X, X)} \leq M, \forall t \in[0, T]
$$

and from (3.17), we have 


$$
\begin{aligned}
\left\|\Psi_{3}(t)\right\|_{X} \leq \int_{0}^{t}\left\|S(t-\tau)\left(N\left(\sum_{i=1}^{3} \Psi_{i}(\tau)\right) \mathrm{d} \tau\right)\right\|_{X} \mathrm{~d} \tau \\
\leq M c\left(\int_{0}^{t}\left\|\Psi_{1}(\tau)\right\|_{X} \mathrm{~d} \tau+\int_{0}^{t}\left\|\Psi_{2}(\tau)\right\|_{X} \mathrm{~d} \tau\right) \\
+M c \int_{0}^{t}\left\|\Psi_{3}(\tau)\right\|_{X} \mathrm{~d} \tau
\end{aligned}
$$

Since $\Psi_{1}$ is solution of the system (3.12), then $\Psi_{1}(\tau)=S(\tau) y_{0}$ and we have

$$
\int_{0}^{t}\left\|\Psi_{1}(\tau)\right\|_{X} \mathrm{~d} \tau \leq M T\left\|y_{0}\right\|_{X}
$$

Since $\Psi_{2}$ is solution of the system (3.13), then we have

$$
\Psi_{2}(\tau)=\int_{0}^{\tau} S(\tau-s)<\varphi_{r}(s), f>_{L^{2}(D)} \chi_{D} f \mathrm{~d} s
$$

and

$$
\begin{aligned}
\left\|\Psi_{2}(\tau)\right\|_{X} & \leq M\|f\|_{L^{2}(D)}^{2} \int_{0}^{\tau}\left\|\varphi_{r}(s)\right\|_{X} \mathrm{~d} s \\
& \leq M^{2} T\|f\|_{L^{2}(D)}^{2}\left\|\varphi_{0}\right\|_{G}
\end{aligned}
$$

then

$$
\int_{0}^{t}\left\|\Psi_{2}(\tau)\right\|_{X} \mathrm{~d} \tau \leq M^{2} T^{2}\|f\|_{L^{2}(D)}^{2}\left\|\varphi_{0}\right\|_{G}
$$

thus

$$
\begin{aligned}
\left\|\Psi_{3}(t)\right\|_{X} \leq & M^{2} c T\left\|y_{0}\right\|_{X}+M^{3} c T^{2}\|f\|_{L^{2}(D)}^{2}\left\|\varphi_{0}\right\|_{G} \\
& +M c \int_{0}^{t}\left\|\Psi_{3}(\tau)\right\|_{X} \mathrm{~d} \tau
\end{aligned}
$$

$$
\begin{aligned}
\left\|B_{1}\right\|_{X} & \leq \int_{0}^{t_{1}}\left(\left\|S\left(t_{1}+h-\tau\right)-S\left(t_{1}-\tau\right)\right\| N\left(\sum_{i=1}^{3} \Psi_{i}(\tau)\right) \mathrm{d} \tau \|_{X}\right) \\
& \leq \varepsilon_{1} C \int_{0}^{t_{1}}\left\|\Psi_{1}(\tau)\right\|_{X} \mathrm{~d} \tau+\epsilon_{1} C \int_{0}^{t_{1}}\left\|\Psi_{2}(\tau)\right\|_{X} \mathrm{~d} \tau+\varepsilon_{1} c \int_{0}^{t_{1}}\left\|\Psi_{3}(\tau)\right\|_{X} \mathrm{~d} \tau
\end{aligned}
$$

Hence, $K_{r}\left(B_{p}\right)$ is uniformly bounded.

Let show that $K_{r}\left(B_{p}\right)$ is relatively compact, indeed: for $0<t_{1}<T$ and $h>0$, we have

$$
\begin{aligned}
& \Psi_{3}\left(t_{1}+h\right)-\Psi_{3}\left(t_{1}\right) \\
& =\int_{0}^{t_{1}}\left(S\left(t_{1}+h-\tau\right)-S\left(t_{1}-\tau\right)\right) N\left(\sum_{i=1}^{3} \Psi_{i}(\tau)\right) \mathrm{d} \tau \\
& +\int_{t_{1}}^{t_{1}+h} S\left(t_{1}+h-\tau\right) N\left(\Psi_{1}(\tau)+\Psi_{2}(\tau)+\Psi_{3}(\tau)\right) \mathrm{d} \tau \\
& =B_{1}+B_{2}
\end{aligned}
$$

where

$$
B_{1}=\int_{0}^{t_{1}}\left(S\left(t_{1}+h-\tau\right)-S\left(t_{1}-\tau\right)\right) N\left(\sum_{i=1}^{3} \Psi_{i}(\tau)\right) \mathrm{d} \tau
$$

and

$$
B_{2}=\int_{t_{1}}^{t_{1}+h} S\left(t_{1}+h-\tau\right) N\left(\Psi_{1}(\tau)+\Psi_{2}(\tau)+\Psi_{3}(\tau)\right) \mathrm{d} \tau
$$

For all $\epsilon_{1}>0$, there exists $\alpha>0$ such that $|h|<\alpha$

$$
\Rightarrow\left\|S\left(t_{1}+h-\tau\right)-S\left(t_{1}-\tau\right)\right\|_{L(X, X)} \leq \epsilon_{1}, \forall \tau \in[0, T]
$$

which gives 
and

$$
A_{2}=c_{1} M c\left[\left(M\left\|y_{0}\right\|_{X}+M^{2} T p\|f\|_{L^{2}(D)}^{2}\right)\left(1+M c T \mathrm{e}^{M c T}\right)\right]
$$

For $\varepsilon_{1} \leq \frac{\varepsilon}{2 A_{1}}$ and $\alpha \leq \operatorname{Inf}\left(\operatorname{dist}\left(B_{p}, C \Omega\right) ; \frac{\varepsilon}{2 A_{2}}\right)$, we obtain $\| P\left(\Psi_{3}\left(t_{1}+h\right)-P\left(\Psi_{3}\left(t_{1}\right) \|_{G^{*}} \leq \varepsilon\right.\right.$. Then, $K_{r}\left(B_{p}\right)$ is relatively compact.

Finally, by the Arzelà-Ascoli theorem see $[29,30]$, $K_{r}: B_{p} \rightarrow G^{*}$ is a compact operator, then $\tilde{K}_{r}: B_{p} \rightarrow G$ is also compact.

Step 2: From (3.16) and (3.20), we have

$$
\begin{aligned}
\left\|\tilde{K}_{r}\left(\varphi_{0}\right)\right\|_{G} \leq & \left\|\wedge^{-1}\left(\chi_{\omega}^{*} y_{d}\right)-\wedge^{-1} P\left(\Psi_{1}(T)\right)\right\|_{G} \\
& +\left\|\wedge^{-1} K_{r}\left(\varphi_{0}\right)\right\|_{G} \\
\leq & A_{3}+M T A_{4}\left\|\varphi_{0}\right\|_{G}
\end{aligned}
$$

where

$$
A_{3}=\left\|\wedge^{-1}\left(\chi_{\omega}^{*} y_{d}\right)-\wedge^{-1} P\left(\Psi_{1}(T)\right)\right\|_{G}+A_{4}
$$

and

$$
A_{4}=\left\|\wedge^{-1}\right\|_{L\left(G^{*}, G\right)} C_{1} M^{2} C T\|f\|_{L^{2}(D)}^{2} \mathrm{e}^{M c T}
$$

The constant $c$ verify

$$
\left\|\wedge^{-1}\right\|_{L\left(G^{*}, G\right)} C_{1} M^{2} C T\|f\|_{L^{2}(D)}^{2} \mathrm{e}^{M c T} \leq \frac{1}{2} .
$$

It is being used the fact that $c(\Omega, T)>0$ is small.

Let $0<s \leq p$ such that $s \geq 2 A_{3}$, then we have $\left\|\tilde{K}_{r}\left(\varphi_{0}\right)\right\|_{G} \leq s \quad \forall \varphi_{0} \in G$ such that $\left\|\varphi_{0}\right\|_{G} \leq s$.

Hence, by applying Schauder's fixed point theorem see [30], the operator $\tilde{K}_{r}$ at least one fixed point, and the proof is completed.

\section{Algorithm 2}

With the same hypothesis as in the last section, we have the following algorithm

- Step 1: we choose the initial conditions, subregion $\Gamma, \omega_{r}, y_{d}$, and the function $f, D$ and $\varepsilon$.

- Step 2: using the pseudo-code.

- Resolution of (3.8) and obtaining $\varphi_{r}$.

- Resolution of (3.12), (3.13) and (3.14)

- Calculation of $\wedge^{-1}$ and obtaining $\tilde{K}_{r}\left(\varphi_{0}\right)$.

- Resolution of $\varphi_{0}=K_{r}\left(\varphi_{0}\right)$ and obtaining $\varphi_{0}$.

- Until $\left\|\varphi_{0}-K_{r}\left(\varphi_{0}\right)\right\| \leq \varepsilon$.

- Step 3: The control $u_{r}^{*}(t)=\left\langle\varphi_{r}(t), f\right\rangle_{L^{2}(D)}$.

\subsection{Numerical Example}

In this subsection, we present a numerical example which illustrate the previous algorithm. It shows that there exists a link between the subregion area and the reached state error, the results are related to the choice of the subregion and the desired state to be reached.

Consider the two-dimensional diffusion system

$$
\begin{aligned}
& \int \frac{\partial y}{\partial t}(x, t)=\frac{\partial^{2} y}{\partial x^{2}}(x, t)+B u(t) \\
& \left.+\sum_{i, j=1}^{\infty}\left|\left\langle y, \varphi_{i j}\right\rangle\right|\left\langle y, \varphi_{i j}\right\rangle \varphi_{i j} \quad \Omega \times\right] 0, T[ \\
& \left.\frac{\partial y}{\partial v_{A}}(0, t)=\frac{\partial y}{\partial v_{A}}(1, t)=0 \quad\right] 0, T[ \\
& y(x, 0)=0
\end{aligned}
$$

\subsubsection{Zone Actuator}

We consider

- The actuator is located in

$$
D=[0.36,0.57] \times[0.38,0.58] \text {. }
$$

- $\Omega=[0,1] \times[0,1], \omega_{r}=[0,0.1] \times[0,0.1]$ : Intern subregion target.

- $\Gamma=\{0\} \times[0,0.4]$ : Boundary subregion target.

- $z_{d}(x)=\left(\frac{1}{6} y^{3}-\frac{1}{2} y^{2}+0.03\right):$ The desired state to be reached in $\Gamma$.

- $y_{d}(x)=\left(\frac{1}{6} x^{3}-\frac{1}{6} x^{2}-0.09\right)\left(\frac{1}{6} y^{3}-\frac{1}{2} y^{2}+0.03\right):$ The extension of desired state $y_{d}$ on $\omega_{r}$.

- Using the previous algorithm 2 in the case zone actuator we have Figures 5-8.

Using the previous algorithm, the regional desired state $y_{d}$ is obtained with error

$$
\left\|\chi_{\Gamma} \gamma_{0} y_{u_{r}^{*}}(T)-y_{d}\right\|_{L^{2}(\Gamma)}^{2}=1.013 \times 10^{-4},
$$

and cost $\left\|u_{r}^{*}\right\|=1.03 \times 10^{-5}$.

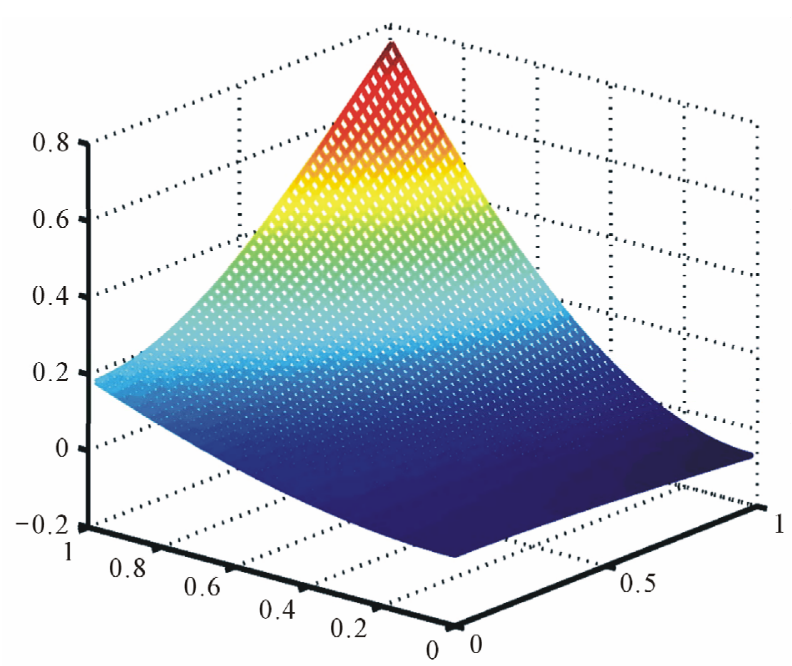

Figure 5. Desired state on the region $\omega_{r}$. 


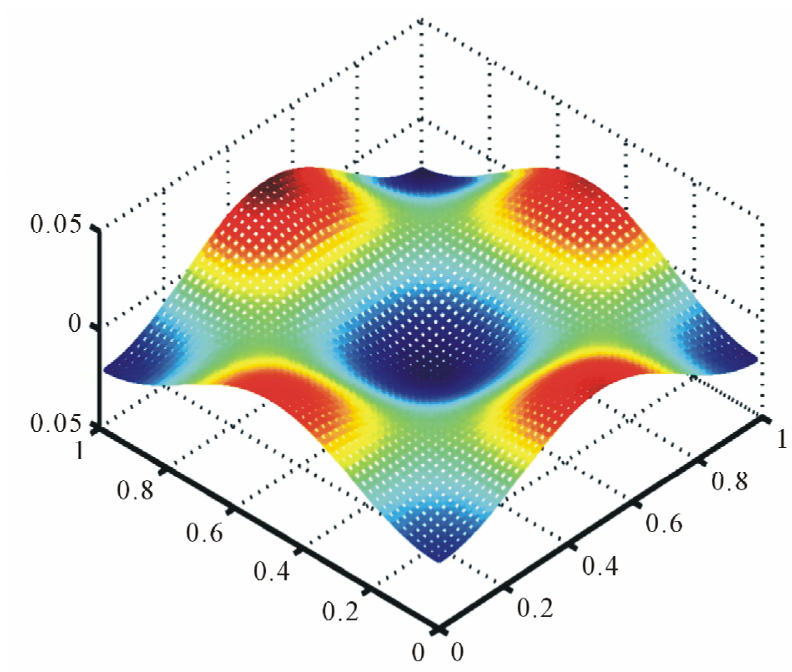

Figure 6. Final state on the region $\omega_{r}$.

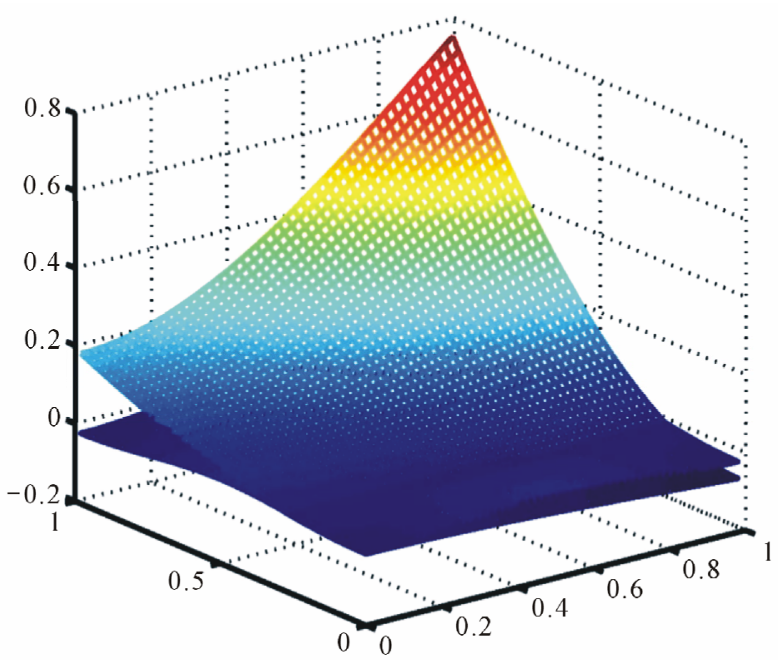

Figure 7. Desired and final state on the region $\omega_{r}$.

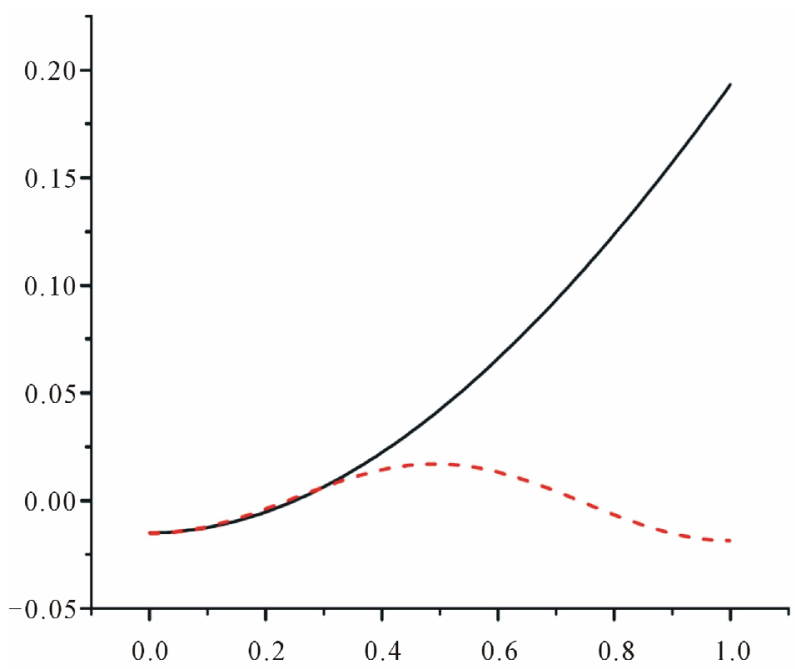

Figure 8. Trace of desired and final state on the region $\Gamma$.

\subsubsection{Relation between the Subregion Area and Reached State Error}

The reached state error depends on the area of the subregion where the desired state has to be given. This error grows with the subregion area. It means that the larger the region is, the greater the error is (see Table 1).

The results are similar for other types of actuator.

\subsubsection{Pointwise Actuator}

In this case, we have

- The actuator is located in $b=\left(b_{1}, b_{2}\right)$ with $b_{1}=$ $0.162, b_{2}=0.165$.

- $\Omega=[0,1] \times[0,1], \omega_{r}=[0,0.2] \times[0,0.5]$ Intern subregion target.

- $\Gamma=\{0\} \times[0,0.5]:$ Boundary subregion target.

- $z_{d}(x)=\left(\frac{2}{3} y^{3}-\frac{1}{5} y^{2}+0.1\right):$ The desired state to be reached in $\Gamma$.

- $y_{d}(x)=\left(\frac{2}{3} x^{3}-\frac{1}{5} x^{2}+0.1\right)\left(\frac{2}{3} y^{3}-\frac{1}{5} y^{2}+0.1\right):$ The extension of desired state $y_{d}$ on $\omega_{r}$.

- Using the previous algorithm 2 in the case point wise actuator we have Figures 9-12.

Table 1. The relation between the subregion area and reached state error.

\begin{tabular}{ll}
\hline Region $\Gamma$ & $\left\|y_{d}-y_{f}\right\|_{L^{2}(\Gamma)}^{2}$ \\
\hline$\{0\} \times[0,0.9]$ & $6.4754 \times 10^{-4}$ \\
$\{0\} \times[0,0.8]$ & $4.6780 \times 10^{-4}$ \\
$\{0\} \times[0,0.6]$ & $2.2452 \times 10^{-4}$ \\
$\{0\} \times[0,0.5]$ & $1.5075 \times 10^{-4}$ \\
$\{0\} \times[0,0.4]$ & $1.0133 \times 10^{-4}$ \\
\hline
\end{tabular}

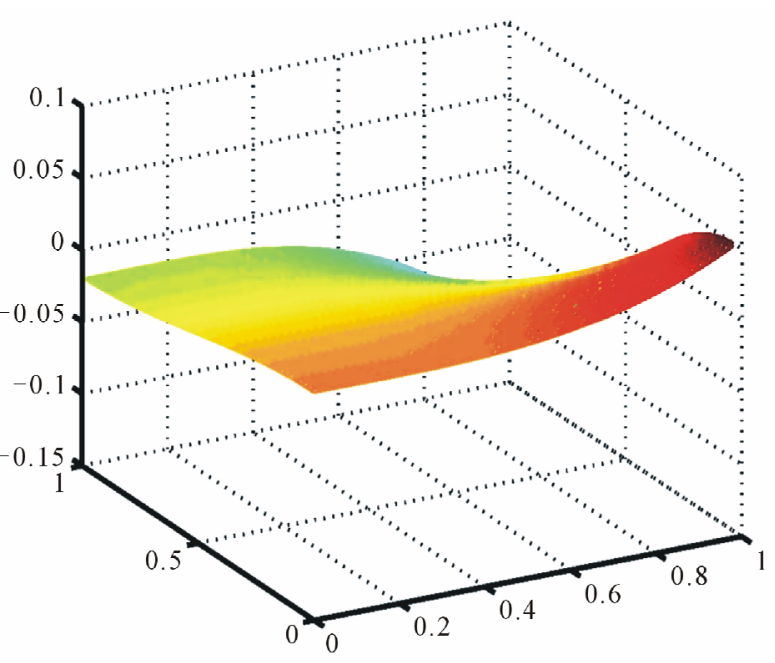

Figure 9. Desired state on the region $\omega_{r}$ 


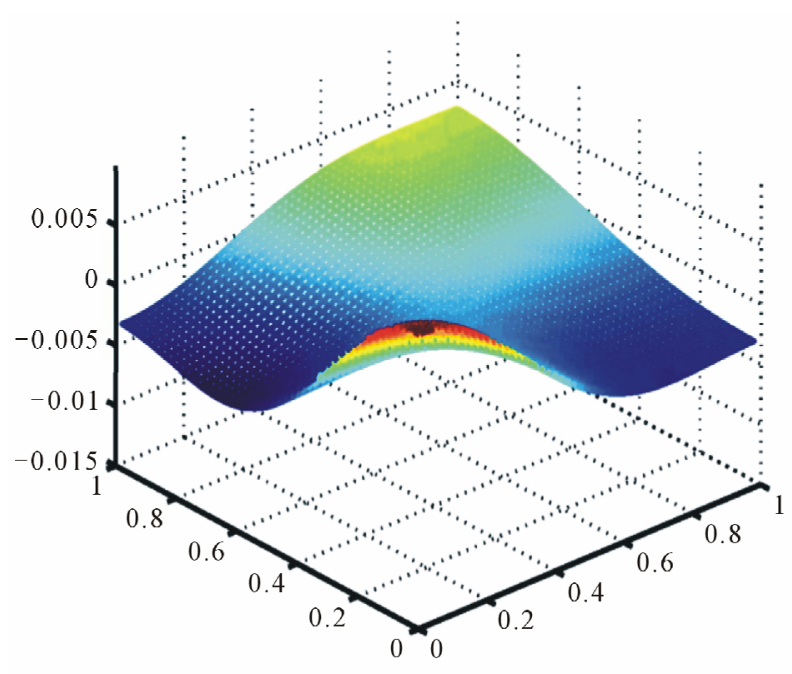

Figure 10. Final state on the region $\omega_{r}$.

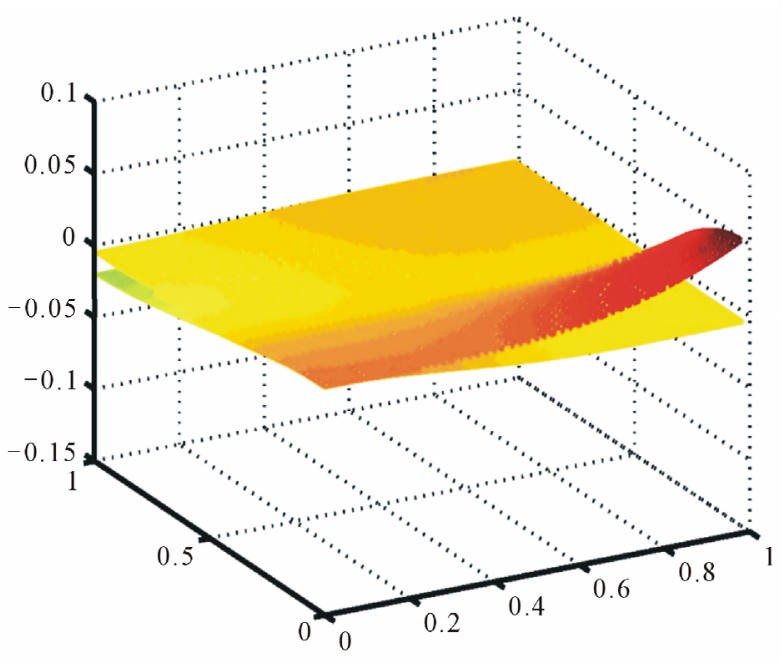

Figure 11. Desired and final state on the region $\omega_{r}$.

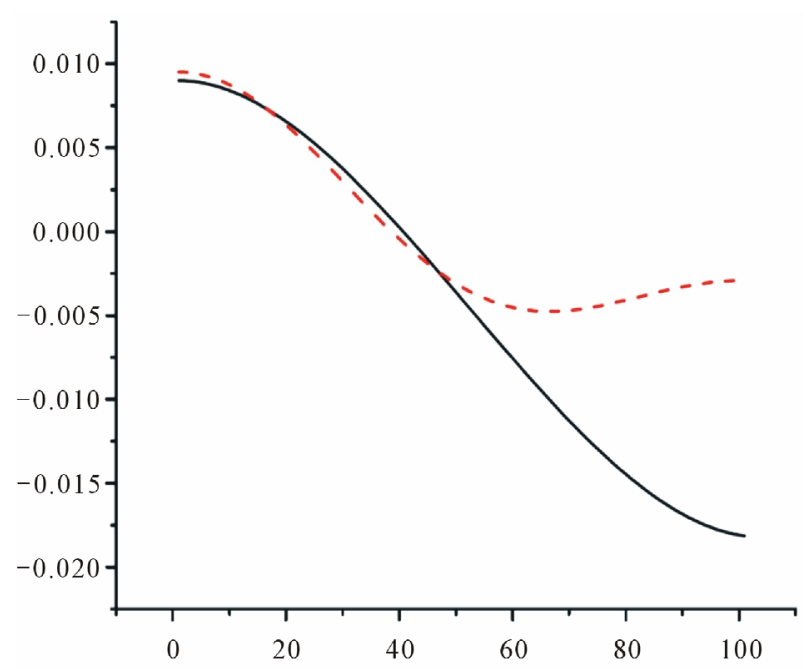

Figure 12. Trace of desired and final state on the region $\Gamma$.

\section{Conclusions}

The work is provide an interesting tool to achieve regional internal and boundary target for a semi-linear parabolic system excited by actuator. The problems of regional controllability are solved using linear regional controllability techniques and by applying HUM method and fixed point theorems. The obtained result leads to an algorithm which was implemented numerically. Examples of various situations and simulations are given.

Various open questions are still under consideration. For example, this is the case of the problem where we test this algorithm for real applications. This case is presently being studied and the results will appear in a separate paper.

The problem of regional controllability problem for semi-linear parabolic systems with time delays is of great interest and the work is under consideration and will be the subject of the feature paper.

\section{REFERENCES}

[1] R. F. Curtain and A. J. Pritchard, "Infinite-Dimensional Linear Systems Theory, Lecture Notes in Control and Information Sciences," Springer Verlag, Berlin, 1978.

[2] R. F. Curtain and H. Zwart, "An Introduction to Infinite Dimensional Linear Systems Theory," Springer Verlag, Berlin, 1995.

[3] K. Balachandran and J. P. Dauer, "Controllability of Nonlinear Systems via Fixed-Point Theorems," Journal of Optimization Theory and Applications, Vol. 53, No. 3, 1987, pp. 345-352. doi:10.1007/BF00938943

[4] H. Zhou, "Approximate Controllability for a Class of Semilinear Abstract Equations," SIAM Journal on Control and Optimization, Vol. 21, No. 4, 1983, pp. 551-555. doi: $10.1137 / 0321033$

[5] K. Naito, "Approximate Controllability for Trajectories of Semilinear Control Systems," Journal of Optimization Theory and Applications, Vol. 60, No. 1, 1989, pp. 57-65. doi:10.1007/BF00938799

[6] X. Li and J. Yong, "Optimal Control Theory for InfiniteDimension a Systems," Birkhauser, Basel, 1994.

[7] W. M. Bian, "Constrained Controllability of Some Nonlinear Systems," Applicable Analysis, Vol. 72, No. 1-2, 1999, pp. 57-73. doi:10.1080/00036819908840730

[8] N. Carmichael and M. D. Quinn, "Fixed-Point Methods in Nonlinear Control, Lecture notes in Control and Information Sciences," Springer Verlag, Berlin, 1984.

[9] X. Zhang, "Exact Controllability of Semilinear Evolution Systems and Its Application," Journal of Optimization Theory and Applications, Vol. 107, No. 2, 2000, pp. 415432. doi:10.1023/A:1026460831701

[10] T. I. Seidmann, "Invariance of the Reachable Set under Nonlinear Perturbations," SIAM Journal on Control and Optimization, Vol. 25, No. 5, 1985, pp. 1173-1191. doi: $10.1137 / 0325064$

[11] J. Klamka, "Constrained Approximate Controllability," 
IEEE Transactions on Automatic Control, Vol. 45, No. 9, 2000, pp. 1745-1749. doi:10.1109/9.880640

[12] J. Klamka, "Schauder's Fixed-Point Theorem in Nonlinear Controllability Problems," Control and Cybernetics, Vol. 29, No. 1, 2000, pp. 153-165.

[13] J. Klamka, "Constrained Controllability of Semilinear Systems," Nonlinear Analysis, Vol. 47, No. 6, 2001, pp. 2939-2949. doi:10.1016/S0362-546X(01)00415-1

[14] K. Balachandran and R. Sakthivel, "Controllability of Integrodifferential Systems in Banach Spaces," Applied Mathematics and Optimization, Vol. 118, No. 1, 2001, pp. 63-71. doi:10.1016/S0096-3003(00)00040-0

[15] C. Fabre, J. P. Puel and E. Zuazua, "Approximate Controllability of the Semilinear Heat Equation", Proceedings of the Royal Society of Edinburgh: Section A Mathematics, Vol. 125, No. 1, 1995, pp. 31-64. doi:10.1017/S0308210500030742

[16] E. Zuazua, "Contrôlabilité Exacte d'un Modèle de Plaques Vibrantes en un Temps Arbitrairement Petit," Comptes Rendus de l'Académie des Sciences, Vol. 304, No. 7, 1987, pp. 173-176.

[17] I. Lasiecka and R. Triggiani, "Exact Controllability of Semilinear Abstract Systems with Applications to Waves and Plates Boundary Control Problems," Applied Mathematics and Optimization, Vol. 23, No. 1, 1991, pp. 109154. doi:10.1007/BF01442394

[18] K. Kassara and A. El Jai, "Algorithme Pour la Commande d'une Classe de Systèmes à Paramètres Répartis non Linéaires," Applied Mathematics and Optimization, Vol. 1, 1993, pp. 3-24.

[19] A. El Jai, A. J. Pritchard and E. Zerrik, "Regional Controllability of Distributed Systems," International Journal of Control, Vol. 62, No. 6, 1995, pp. 1351-1365. doi: $10.1080 / 00207179508921603$
[20] E. Zerrik, A. Boutoulout and A. El Jai, "Actuators and Regional Boundary Controllability of Parabolic Systems," International Journal of Systems Science, Vol. 31, No. 1, 2000, pp. 73-82. doi:10.1080/002077200291479

[21] J. Jacob, "Modélisation et Simulation Dynamique de Procédés des Eaux de Type Biofiltre. Traitement d'équations Différentielles Partielles Etalgébriques," Thése de Doctorat I. N. P., Toulouse, 1994.

[22] A. El Jai and A. J. Pritchard, "Capteurs et Actionneurs dans l'analyse des Systèmes Distribués," Masson, Paris, 1986.

[23] A. Pazy, "Semigroups of Linear Operators and Applications to Partial Differential Equations," Springer-Verlag, Berlin, 1983.

[24] J. L. Lions and E. Magenes, "Problèmes aux Limites non Homogènes et Applications," Dunod, Paris, 1968.

[25] E. Zerrik and A. Kamal, "Output Controllability for Semi-Linear Distributed Parabolic Systems," Journal of Dynamical and Control Systems, Vol. 13, No. 2, 2007, pp. 289-306. doi:10.1007/s10883-007-9014-8

[26] A. El Jai and A. J. Pritchard, "Sensors and Actuators in Distributed Systems Analysis," Ellis Horwood, Chichester, 1988.

[27] A. El Jai, "Eléments de Contrôlabilité," Presses Universitaires de Perpignan, Perpignan, 2006.

[28] J. L. Lions, "Contrôlabilité Exacte, Perturbations et Stabilisation des Systèmes Distribués," Masson, Paris, 1988.

[29] H. Brezis, "Analyse Fonctionnelle: Théorie et Application," Masson, Paris, 1983.

[30] E. Zeidler, "Applied Functional Analysis: Applications to Mathematical Physics,” Springer-Verlag, Berlin, 1995. 